\title{
Coalescence, un imaginaire scientifique
}

\section{Coalescence, Visions of Science}

\author{
Alexandre Darmon ${ }^{1}$, Julie Simon ${ }^{1}$ \\ ${ }^{1}$ AiR - Art in Research, Paris, darmon@artinresearch.com, julie.simon@artinresearch.com
}

RÉSUMÉ. Coalescence. Inévitable attraction, fusion inéluctable de deux matières identiques qui résulte en la création d'une nouvelle entité, plus efficace énergétiquement. Telle est l'essence de cet ouvrage qui associe science et art au service de la recherche du beau. Révélant l'esthétique saisissante des nombreuses formes de la nature, les photographies du livre Coalescence, un imaginaire scientifique subliment la recherche scientifique et rappellent à quel point la nature peut être belle et déconcertante. Saisis par les chercheurs, ces instantanés de beauté déroutent et font travailler l'imaginaire ; ils sont autant emplis de mystère qu'empreints d'une indiscutable vérité.

ABSTRACT. Coalescence. Inevitable attraction, inescapable fusion of two identical materials that results in the creation of a new, more energy-efficient entity. This is the essence of this work which associates science and art in a quest for beauty. Revealing the striking aesthetics of the many forms of nature, the photographs of the book Coalescence, Visions of Science enhance scientific research and remind us how beautiful and disconcerting nature can be. Captured by researchers in their laboratory, these moments of beauty spark the imagination. Although filled with a sense of mystery, they expose an indisputable truth.

MOTS-CLÉS. Coalescence, Science, Art, Photographie, Recherche, Imaginaire, Nature.

KEYWORDS. Coalescence, Science, Art, Photography, Research, Imaginary, Nature.

\section{$\underline{\text { Coalescence }}$}

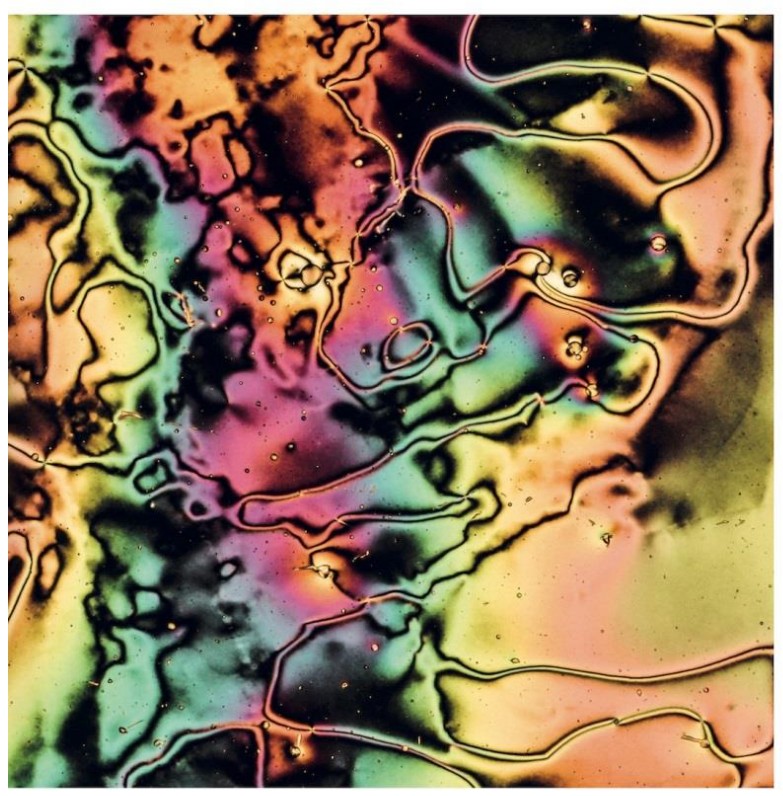

Un imaginaire scientifique

Visions of Science

Art in Research

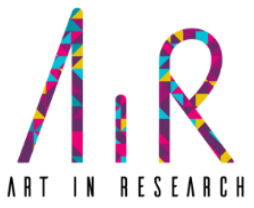


Coalescence : inévitable attraction, fusion inéluctable de deux matières identiques qui résulte en la création d'une nouvelle entité, plus efficace énergétiquement. Telle est l'essence de cet ouvrage qui associe science et art au service de la recherche du beau. Révélant l'esthétique saisissante des nombreuses formes de la nature, les photographies du livre Coalescence, un imaginaire scientifique subliment la recherche scientifique et rappellent à quel point la nature peut être belle et déconcertante. Saisis par les chercheurs, ces instantanés de beauté déroutent et font travailler l'imaginaire ; ils sont autant emplis de mystère qu'empreints d'une indiscutable vérité.

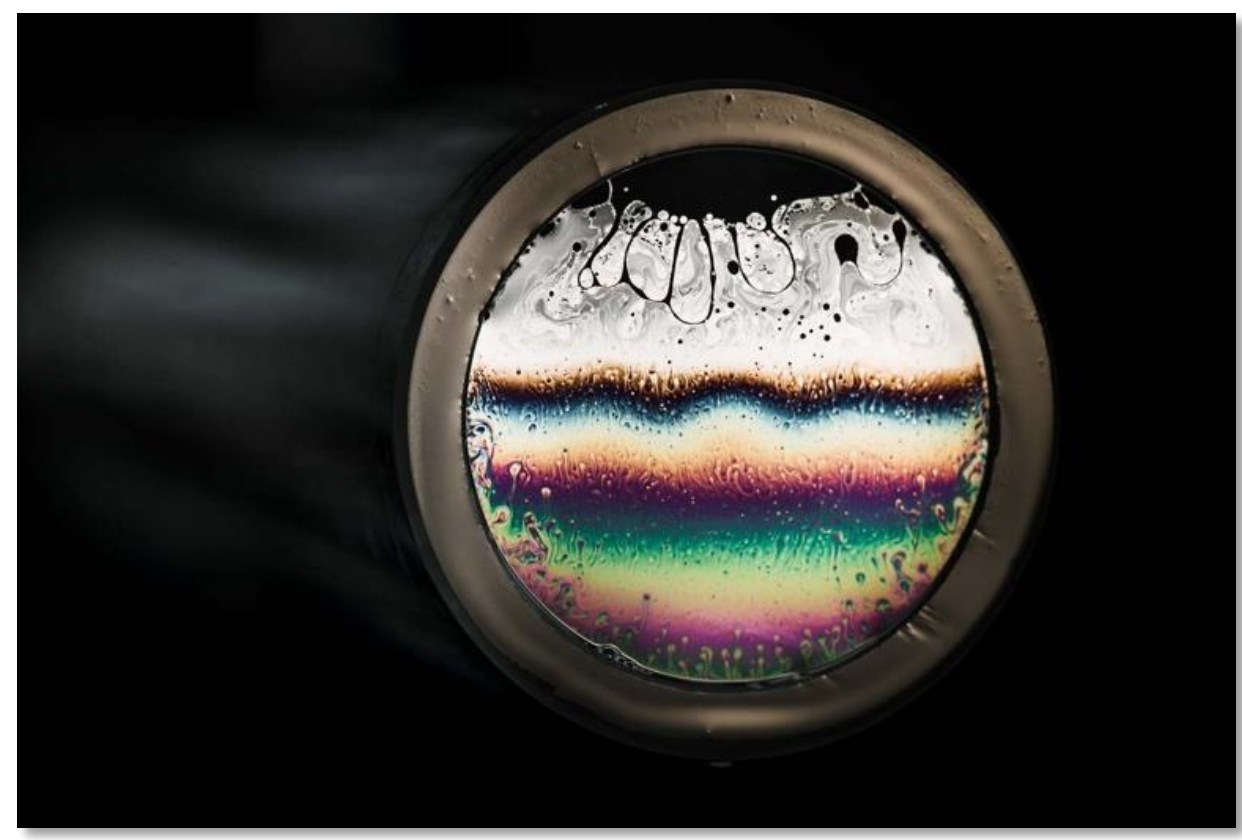

Un instant de répit

Florence Elias

Consciente que la science est emplie d'une beauté stupéfiante, AiR - Art in Research s'est donnée pour mission de révéler au plus grand monde le travail de la recherche scientifique en y intégrant une dimension artistique à la croisée du réel et de l'imaginaire. En regroupant près de 70 images, principalement des photographies, de plus d'une quinzaine de chercheuses et de chercheurs français, Coalescence, un imaginaire scientifique dévoile à tous un aperçu de l'infinie beauté de la recherche scientifique. L'ambition d'un tel projet est de mettre en avant le travail que portent les scientifiques sur leur travail en proposant des tirages d'art de photographies prises par les chercheurs dans leur laboratoire.

L'utilisation de la photographie est aujourd'hui prépondérante dans bien des domaines scientifiques : médecine, biologie, physique, chimie, sismologie... Véritable outil d'investigation scientifique, les chercheurs l'emploient aussi bien pour l'interprétation que pour le partage de l'information. Il s'agit en vérité d'une réelle passion qu'ils décident de transmettre par le biais des images aussi fascinantes soient-elles. En enfilant le costume d'artiste, les scientifiques contribuent ainsi à révéler la splendeur des phénomènes qu'ils observent et dévoilent de la plus belle des façons la dimension artistique de la science. 


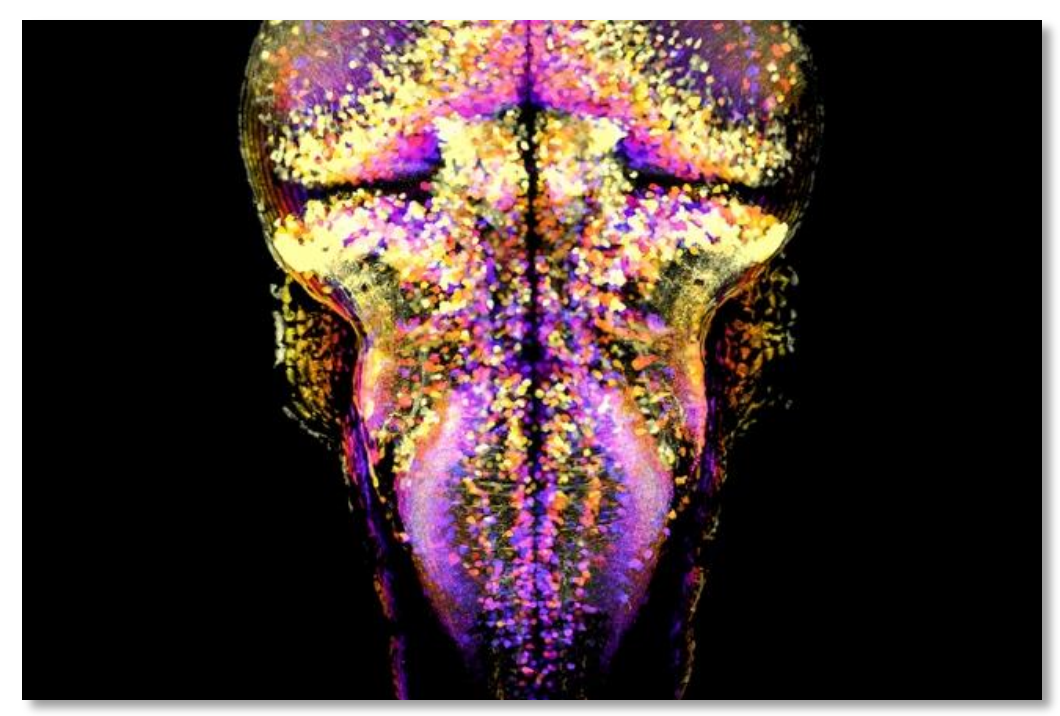

Neurones en lumières I

Claire Wyart \& Kristen Severi

La collection de photographies de Coalescence, un imaginaire scientifique s'articule autour des thèmes de la vie et du jeu d'échelle. Les prises de vue, tantôt microscopiques, tantôt macroscopiques, jouent avec les perceptions du spectateur, plongé dans un univers aux échelles nouvelles. Ce dernier y découvre les formes insoupçonnées de la nature évoluant dans un monde infiniment grand et puissant, en proie à une incroyable complexité. C'est notamment cette suprématie de l'univers qui attise depuis toujours la curiosité des chercheurs fascinés par la capacité de la science à répondre aux questions qui les submergent.

"Le travail de AiR nous rappelle, mieux que tout autre, que la nature est la plus grande des artistes." Cédric Villani, Médaille Fields

Les photographies présentes dans ce livre stimulent l'imaginaire et invitent le spectateur à s'immiscer dans un monde irréel. L'ouvrage permet de laisser libre cours à son imagination et d'interpréter de façon totalement insouciante la beauté de la nature qui se révèle sous nos yeux. Il s'agit là de véritablement plonger, le temps d'un instant, celui qui admire les images dans un univers aux échelles dérisoires, souvent imperceptible à l'œil nu. L'expérience du spectateur est notamment prolongée par les textes qui accompagnent chacune des œuvres : autant poétiques qu'explicatifs, les descriptifs sont là pour prolonger la rêverie et donner quelques clés de lecture scientifique.

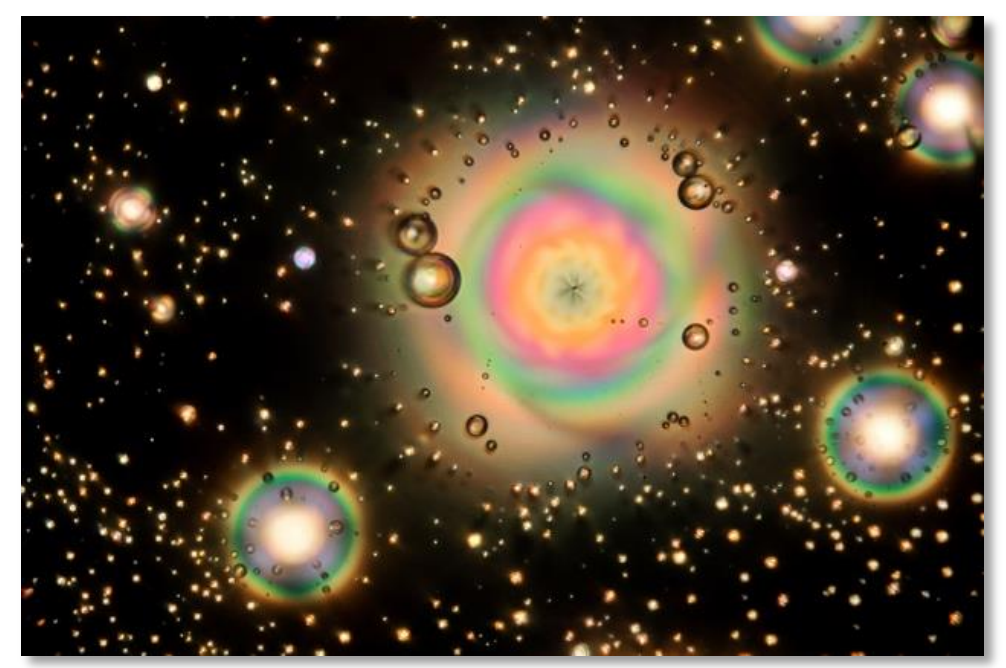




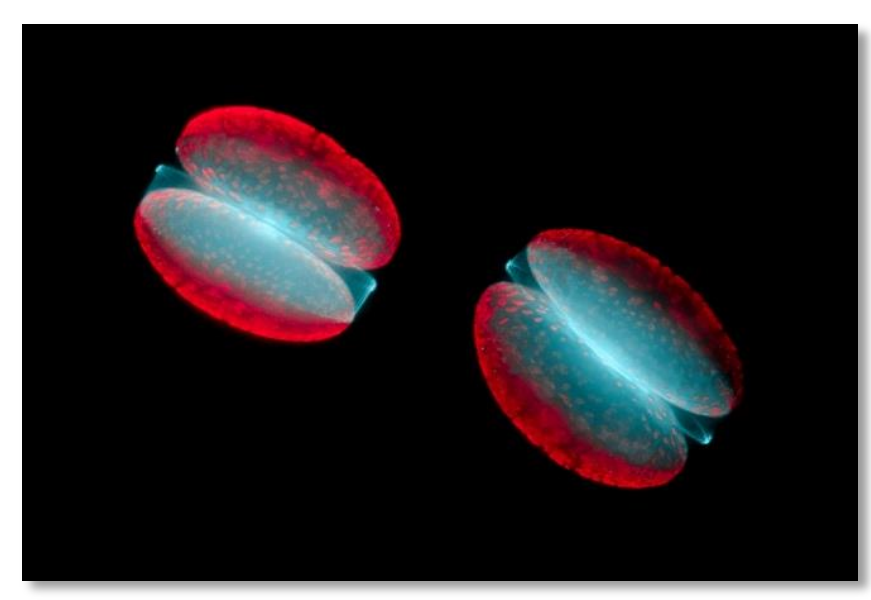

«Diatomées de tous les pays, unissez-vous ! Soyez fécondes et multipliez-vous, remplissez la mer ! Ce couple de microalgues unicellulaires a pris au pied de la lettre cette très vieille injonction et se retrouve surpris à un moment critique, celui de la division cellulaire. Simultanément, ces deux bourgeons pellucides donnent corps à des copies parfaitement identiques d'elles-mêmes. En bleu, le squelette en silice va lui aussi bientôt se fractionner et chacune des moitiés migrer avec la cellule fille en rouge. Pour ces diatomées, c'est le début d'une nouvelle vie, d'une nouvelle ère. »

Diatomées, mère et fille III

Pascal-Jean Lopez

Le livre Coalescence, un imaginaire scientifique est le travail de plus d'un an de réflexion et d'immersion dans les laboratoires de recherche dont l'ambition est de révéler au monde la beauté cachée des sciences de la nature. Alliant réel et imaginaire, les photographies incitent le spectateur à voyager dans un monde aux échelles déroutantes et à interpréter à sa façon les images qu'il perçoit. Plus qu'un livre scientifique, il s'agit là d'un véritable ouvrage d'art qui interpelle et interroge les plus curieux sur les mystères de la science.

Un article sur le thème de la photographie scientifique paraîtra prochainement dans la revue.

\section{Référence}

ART IN RESEARCH, Coalescence, un imaginaire scientifique, LIENART édition, 2019, 127 p. ISBN : 978-2-35906280-9. 